\title{
On Applicability of the Kutta-Joukowski Theorem to Low- Reynolds-Number Unsteady Flows
}

\author{
Shizhao Wang ${ }^{1}$, Xing Zhang ${ }^{2}$ and Guowei $\mathrm{He}^{3}$ \\ LNM, Institute of Mechanics, Chinese Academy of Sciences, Beijing 100190, China \\ and \\ Tianshu $\mathrm{Liu}^{4}$ \\ Department of Mechanical and Aeronautical Engineering, Western Michigan University, Kalamazoo, MI 49008
}

\begin{abstract}
The limitations of the Kutta-Joukowski (K-J) theorem in prediction of the time-averaged and instantaneous lift of an airfoil and a wing in low-Reynolds-number unsteady flows are examined. A general lift formula for a rectangular control volume is given in a very simple form in the framework of viscous flow theory, which provides a rational foundation for a direct comparison with the $\mathrm{K}-\mathrm{J}$ theorem considered as a reduced case. Direct numerical simulations on the stationary and flapping flat plate and rectangular wing are conducted to assess the accuracy of both the K-J theorem and the general lift formula. In particular, the Lamb vector integral for the vortex force and the acceleration term of fluid for the unsteady inertial effect are evaluated as the main contributions to the unsteady lift generation of a flapping wing.
\end{abstract}

\section{Nomenclature}

$\begin{array}{lll}A & =\text { flapping amplitude } \\ f & =\text { flapping frequency } \\ \mathbf{F} & =\text { aerodynamic force } \\ \overline{C_{l}} & =\text { time-average lift coefficient } \\ L & =\text { lift } \\ L^{\prime} & =\text { lift per unit span } \\ \boldsymbol{n} & =\text { unit normal vector pointing to the outside of the control volume } \\ p & =\text { pressure } \\ \boldsymbol{u} & =\text { velocity of fluid } \\ u_{e f f} & =\text { effective velocity } \\ U_{\infty} & =\text { velocity of upstream flow } \\ V_{f} & =\text { control volume of the fluid } \\ \alpha & =\text { geometry angle of attack } \\ \Gamma & =\text { circulation around the foil } \\ \rho & =\text { density of fluid } \\ \Sigma & =\text { control surface } \\ \tau & =\text { surface shear stress vector } \\ \boldsymbol{\omega} & =\text { vorticity } \\ \partial B & =\text { solid boundary of a body }\end{array}$

${ }^{1}$ Assistant professor, Institute of Mechanics, Chinese Academy of Sciences.

${ }^{2}$ Associate professor, Institute of Mechanics, Chinese Academy of Sciences.

${ }^{3}$ Professor, Institute of Mechanics, Chinese Academy of Sciences, Email: hgw@1nm.imech.ac.cn

${ }^{4}$ Professor, Department of Mechanical and Aeronautical Engineering, Western Michigan University.

American Institute of Aeronautics and Astronautics 


\section{Introduction}

$\mathrm{L}$ OW-Reynolds-number flight particularly flapping flight has recently attracted considerable attention in the aeronautical communities due to the need of developing biologically-inspired micro air vehicles (MAV) ${ }^{1,2}$. Therefore, animal flight, which has been traditionally studied by avian zoologists, becomes immediately relevant to this engineering research. Natural flyers include birds, insects and bats. In bird flight, the Reynolds numbers based on the mean wing chord range from $10^{4}$ to $10^{6}$ while the Reynolds numbers for bat flight are $10^{3}-10^{5}$. In contrast, the Reynolds numbers for insects are typically less than 5000. To understand unsteady flow fields around flying birds and bats, particle image velocimetry measurements have been conducted in wind tunnels ${ }^{3-7}$. Since the aerodynamic forces of a flying animal cannot be directly measured using a force balance, the Kutta-Joukowski (K-J) theorem has been used in these studies to infer the lift after the circulation is estimated by integrating the vorticity field in a selected cross-section region near wings or in wakes generated in flapping flight. The K-J theorem gives the lift per unit span on an airfoil by $L^{\prime}=\rho U_{\infty} \Gamma$, where $\Gamma$ is the circulation around the airfoil, $U_{\infty}$ is the incoming flow velocity, and $\rho$ is the fluid density. Then, integration of $L^{\prime}$ along an effective span provides the total lift of a flying animal. Clearly, the distinct advantage of using the K-J theorem is its simplicity, which is directly related to vorticty fields that can be measured by PIV. In particularly, it allows estimation of the lift components contributed by certain distinct vortical structures such as the leading-edge vortices. On the other hand, to calculate the lift, the K-J theorem has served as an essential mechanism in vortex-based aerodynamics models for low-Reynolds-number flapping flight ${ }^{3,8-12}$. The classical aerodynamics models have been adapted further by incorporating some relevant flow phenomena like the leading-and trailing-edge vortices for additional lift generation $^{13,14}$.

However, lift estimation using the K-J theorem based on PIV measurements in the wakes of slowly-flying birds (pigeons and jackdaws) gave a significantly lower value of the lift that cannot support the bird weight, which is called "the wake momentum paradox" by Spedding et al. ${ }^{3}$. The wake momentum paradox is considered presumably as a result of under-resolved vorticity measurements that lead to underestimation of the circulation in complex wakes. Another possibility for this paradox is that certain high-lift-generation mechanisms in low-Reynolds-number flight such as the wake capture in insect flight may exist in slowly-flying birds, but they are not detectable in wake measurements. Recent PIV measurements in the wakes of flying bats by Hubel et al. ${ }^{6,7}$ also indicated that the average circulation was only about one half of the value required for weight support at the observed flight speed. At this stage, the wake momentum paradox remains unanswered. In this work, this problem is viewed from a different perspective, and our attention is paid on the K-J theorem itself. A fundamental question is whether the K-J theorem captures the main lift-generating mechanisms in highly unsteady separated flows at low Reynolds numbers in flapping flight. It is necessary to quantitatively evaluate the accuracy of the K-J theorem when it is applied to lowReynolds-number unsteady flows.

The objectives of this study are two-fold. First, the applicability of the K-J theorem will be discussed in a light of the general viscous flow theory and quantitatively examined through direct comparison with the lift calculated from direct numerical simulations (DNS) on low-Reynolds-number flows over the stationary and flapping twodimensional (2D) flat plate and rectangular flat-plate wing. Further, a general lift formula will be given in a very simple form and validated for more accurate estimation of the unsteady lift. This paper is organized as follows. The K-J theorem is first discussed as a reduced case of the general lift formula derived from the Navier-Stokes equations for a rectangular control volume. The mechanisms neglected in the K-J theorem applied to unsteady flows are identified. The numerical method, the immersed boundary method based on discrete stream function formulation, and its validation are briefly described. Then, the flows over the stationary and flapping flat plate are simulated, and the lift coefficients given by using the K-J theorem and the general lift formula are directly compared with DNS. Finally, the flows over the stationary and flapping rectangular flat-plate wing with the aspect ratio of 4 are simulated to further examine the accuracy of the K-J theorem and the general lift formula in three-dimensional (3D) cases.

\section{Lift Expressions}

\section{A. General lift formula}

In classical aerodynamics, the K-J theorem is derived in the $2 \mathrm{D}$ potential flow over a circular cylinder with a given value of the circulation, and then it is extended to an airfoil via a conformal transformation where the circulation is determined by applying the Kutta condition at the trailing edge ${ }^{15,16}$. Further, in the Prandtl lifting-line theory, the K-J theorem is applied to a bound vortex line along the wing span to model the aerodynamic flow over a finite wing. Although the K-J theorem is originally derived in the framework of the inviscid flow theory, the 
circulation is physically originated from the viscous shearing motion in a boundary layer around an airfoil. Actually, the Kutta condition represents the outcome of the generation of the circulation through viscous shearing. The application of the K-J theorem is well founded for high-Reynolds-number attached flows over airfoils since the flow outside a thin boundary layer can be considered to be inviscid. The circulation can be calculated by integrating the vorticity field in a boundary layer in a suitably large integration domain covering the upper and lower surfaces of an airfoil. However, in flight of small birds, bats and insects, not only the Reynolds numbers are usually much smaller, but also the flow around a flapping thin wing at high angle of attack (AoA) is highly unsteady and separated, generating energetic and organized vortices such as the leading- and trailing-edge vortices. In fact, the lift generated by these vortices in unsteady separated flows is necessary for low-Reynolds number animal flight. In this case, the legitimacy of applying the K-J theorem has to be examined in the framework of the viscous flow theory.

In an incompressible viscous flow, as shown in Fig. 1, the force acting on a solid body is given by

$$
\boldsymbol{F}=-\oint_{\partial B}(-p \boldsymbol{n}+\boldsymbol{\tau}) d S=-\rho \int_{V_{f}} \boldsymbol{a} d V+\oint_{\Sigma}(-p \boldsymbol{n}+\boldsymbol{\tau}) d S
$$

where $p$ is the pressure, $\tau$ is the surface shear stress vector, $\boldsymbol{a}=D \boldsymbol{u} / \mathrm{D} t$ is the acceleration, $\rho$ is the fluid density, $\partial B$ denotes a solid boundary of the body domain $B, V_{f}$ denotes the control volume of fluid, $\Sigma$ denotes an outside control surface in which the body is enclosed, and $\boldsymbol{n}$ is the unit normal vector pointing to the outside of a control surface. However, it is difficult to use Eq. (1) to infer the force in measurements. It is not easy to measure surface pressure and skin friction fields in low-speed flows even though pressure-sensitive paint measurement and global skin friction diagnostics are promising to obtain the integrated forces ${ }^{17,18}$. Therefore, a simple alternative force formula is desirable.

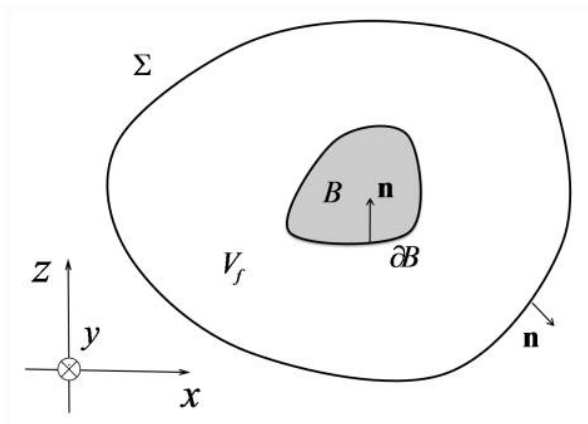

Figure 1. Schematic of a control surface around a body

$$
\begin{gathered}
\text { Substitution of } \boldsymbol{a}=D \boldsymbol{u} / D t=\partial \boldsymbol{u} / \partial t+\boldsymbol{u} \cdot \nabla \boldsymbol{u}=\partial \boldsymbol{u} / \partial t+\boldsymbol{\omega} \times \boldsymbol{u}+\nabla\left(q^{2} / 2\right) \text { into Eq. (1) leads to } \\
\mathbf{F}=\rho \int_{V_{f}} \mathbf{u} \times \boldsymbol{\omega} d V-\rho \int_{V_{f}} \frac{\partial \mathbf{u}}{\partial t} d V-\oint_{\Sigma}\left(p+\rho q^{2} / 2\right) \mathbf{n} d S+\oint_{\Sigma} \tau d S-\rho \oint_{\partial B}\left(q^{2} / 2\right) \mathbf{n} d S,
\end{gathered}
$$

where $\boldsymbol{u}$ is the velocity, $\boldsymbol{\omega}$ is the vorticity, and $q=|\boldsymbol{u}|$. The first term in the right-hand side (RHS) of Eq. (2) is a volume integral of the Lamb vector $\boldsymbol{u} \times \boldsymbol{\omega}$ that represents the vortex force. For convenience, it is simply called the Lamb vector integral. The second term is a volume integral of the local acceleration of fluid for the unsteady inertial effect. The third and fourth terms are the surface integrals of the total pressure of flow and the surface shear stress on the control surface $\Sigma$. The fifth term represents the effect of a moving surface of a body on the force. Since the pressure $p$ in space is very difficult to measure, the third term related to $p$ in Eq. (2) should be transformed to the terms related to the velocity that is measurable. Several general force expressions have been given by $\mathrm{Wu} \& \mathrm{Wu}^{19}$, Noca, Shiells \& $\mathrm{Jeon}^{20}, \mathrm{Wu}, \mathrm{Ma} \& \mathrm{Zhou}^{21}$, and $\mathrm{Wu}, \mathrm{Lu} \&$ Zhuang ${ }^{22}$. However, for a general control surface, eliminating the troublesome pressure term usually leads to more complicated expressions in which the physical meanings and relative contributions of some terms are not easily elucidated. The expressions based on "derivativemoment transformations" of $\mathrm{Wu}$ et $\mathrm{al}^{22}$ provide a better way to investigate the role of local flow structures on the force and moment. However, some major terms, such as $\nabla^{2} \boldsymbol{\omega}$ and $\partial \boldsymbol{\omega} \partial t$, are difficult to measure accurately in experiments since the third-order spatial and second-order mixed derivatives of velocity fields are required.

In this work, we circumvent this problem by selecting a rectangular control volume to obtain a very simple lift formula. Consider a coordinate system $(x, y, z)$ defined by the unit orthogonal vectors $(\boldsymbol{i}, \boldsymbol{j}, \mathbf{k})$ where $\boldsymbol{i}$ and $\mathbf{k}$ are the unit vectors parallel and normal to the freestream, respectively, and $\boldsymbol{j}=\boldsymbol{k} \times \boldsymbol{i}$ is the unit vector along the spanwise direction. The lift on a body is given by $L=F_{z}=\mathbf{k} \cdot \mathbf{F}$. More specifically, as shown in Fig. 2, a rectangular domain $D$ is selected as a control volume to simplify the lift expression. The unit normal vectors on 
the left and right faces (sections) of the boundary $\partial D$ are perpendicular to $\mathbf{k}$, i.e., $\mathbf{k} \cdot \mathbf{n}=0$. Note that for 3D flows the unit vectors on the side faces of the control volume are also perpendicular to $\mathbf{k}$, i.e., $\boldsymbol{j} \cdot \mathbf{n}=0$. Furthermore, if the upper and lower sections of $\partial D$ are located so far away from the body that $p+\rho q^{2} / 2=$ const. (the Bernoulli equation) in the inviscid flow, the integrals on these sections are cancelled out. Therefore, the contribution of the troublesome third term of RHS of Eq. (2) to the lift is zero. For a sufficiently large boundary $\partial D, \mathbf{k} \cdot \tau$ on the most portion of $\partial D$ is zero except in a wake, and thus the contribution of the fourth term in RHS of Eq. (2) to the lift can be neglected.

The fifth term in RHS of Eq. (2) reflects the effect of a moving boundary. If a body is stationary in flow, this term is zero due to the zero-velocity boundary condition. For a moving rigid body, the velocity of a point on the body boundary can be expressed as

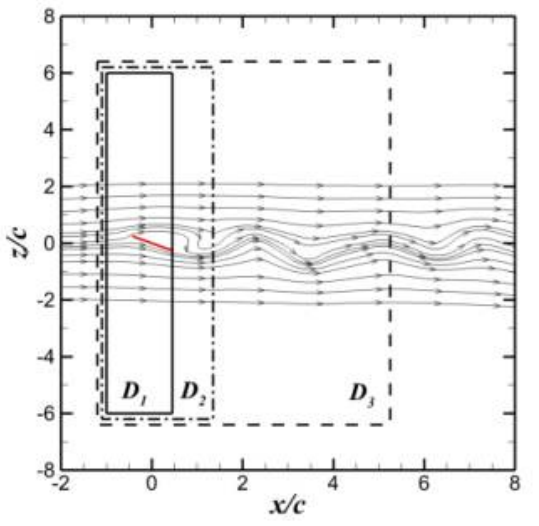

Figure 2. Integral domains enclosing an airfoil or a wing $\mathbf{u}=\mathbf{u}_{c}+\boldsymbol{\Omega} \times \mathbf{r}$, where $\mathbf{u}_{c}$ and $\boldsymbol{\Omega}$ are the translation and angular velocities of the body, respectively, and $\mathbf{r}=\boldsymbol{x}-\boldsymbol{x}_{o}$ is the positional vector from the rotating center to a point in the body. To evaluate the fifth term in RHS of Eq. (2), we use the following relation

$$
\oint_{\partial B}\left(q^{2} / 2\right) \mathbf{n} d S=\int_{B} \nabla\left(q^{2} / 2\right) d V=\left(\mathbf{u}_{c} \times \mathbf{\Omega}\right) V_{B}+(\boldsymbol{\Omega} \cdot \boldsymbol{\Omega})\langle\boldsymbol{r}\rangle_{B} V_{B}-\boldsymbol{\Omega}\left(\boldsymbol{\Omega} \cdot\langle\boldsymbol{r}\rangle_{B}\right) V_{B},
$$

where $V_{B}$ is the volume of the body and $\langle\boldsymbol{r}\rangle_{B}$ is a domain-averaged position vector defined as

$$
\langle\boldsymbol{r}\rangle_{B}=V_{B}^{-1} \int_{B} \mathbf{r} d V
$$

An estimate is $\left|\boldsymbol{u}_{c} \times \boldsymbol{\Omega}\right|=C_{1}\left|\boldsymbol{u}_{c}\right||\boldsymbol{\Omega}|$, where $C_{1}$ is a positive constant. Without loss of generality, another estimate is $\left|(\boldsymbol{\Omega} \cdot \boldsymbol{\Omega})\langle\boldsymbol{r}\rangle_{B}-\boldsymbol{\Omega}\left(\boldsymbol{\Omega} \cdot\langle\boldsymbol{r}\rangle_{B}\right)=C_{2}\right| \boldsymbol{u}_{\text {lin }}|\boldsymbol{\Omega}|$, where $C_{2}$ is a positive coefficient and $\left|\boldsymbol{u}_{\text {lin }}\right|$ is a characteristic linear velocity of the rotating body. To evaluate the fifth term compared to the Lamb vector integral (the first term), we consider the following condition

$$
\frac{u_{\text {char }}|\boldsymbol{\Omega}|}{\left|\langle\boldsymbol{u} \times \boldsymbol{\omega}\rangle_{D}\right|} \frac{V_{B}}{V_{D}}<<1,
$$

where $u_{\text {char }}=C_{1}\left|\boldsymbol{u}_{c}\right|+C_{2}\left|\boldsymbol{u}_{\text {lin }}\right|$ is the total characteristics velocity of the moving body, and $\langle\boldsymbol{u} \times \boldsymbol{\omega}\rangle_{D}$ is the domain-averaged Lamb vector over the domain $D$. Here the domain averaged operator is defined as

$$
\langle\bullet\rangle_{D}=V_{D}^{-1} \int_{V_{D}} \bullet d V
$$

When Eq. (5) holds, the contribution of the fifth term to the lift can be neglected compared to the Lamb integral. Further, an estimate is $\left|\langle\boldsymbol{u} \times \boldsymbol{\omega}\rangle_{D}\right|=C_{3}\left|\langle\boldsymbol{u}\rangle_{D}\right|\left|\langle\boldsymbol{\omega}\rangle_{D}\right|$, where $C_{3}$ is a positive coefficient and $\left|\langle\boldsymbol{\omega}\rangle_{D}\right|$ is proportional to the circulation. Thus, Eq. (5) is re-written as 


$$
\frac{u_{\text {char }}}{\left|\langle\boldsymbol{u}\rangle_{D}\right|} \frac{|\boldsymbol{\Omega}|}{\left|\langle\boldsymbol{\omega}\rangle_{D}\right|} \frac{V_{B}}{V_{D}}<<1 .
$$

For a fixed wing, $u_{\text {char }} /\left|\langle\boldsymbol{u}\rangle_{D}\right|=0$, and for a flapping wing $u_{\text {char }} /\left|\langle\boldsymbol{u}\rangle_{D}\right| \sim O(1)$. It is assumed that the rotational rate $|\boldsymbol{\Omega}|$ of a body is in the same order of $\left|\langle\boldsymbol{\omega}\rangle_{D}\right|$, i.e., $|\boldsymbol{\Omega}| /\left|\langle\boldsymbol{\omega}\rangle_{D}\right| \sim O(1)$. This assumption is supported by DNS on a flapping wing in this work. In other words, the Stokes number is $S t=t_{\omega} / t_{\Omega} \sim O(1)$, where $t_{\Omega}=|\boldsymbol{\Omega}|^{-1}$ is the timescale of a body rotation and $t_{\omega}=\left|\langle\boldsymbol{\omega}\rangle_{D}\right|^{-1}$ is the timescale of the fluid vorticity. For a thin wing, the volume ratio between the wing and the control volume is small, i.e., $V_{B} / V_{D}<<1$, where $V_{D}$ is the volume of the rectangular domain $D$. Hence, Eq. (5) or (7) is a reasonable assumption, and the fifth term in RHS of Eq. (2) can be neglected.

After the higher-order smaller terms are neglected, the contributions of the first and second terms in RHS of Eq. (2) are retained, and as a result the lift is given by a very simple formula

$$
L=\rho V_{D} \boldsymbol{k} \cdot\left(\langle\boldsymbol{u} \times \boldsymbol{\omega}\rangle_{D}-\langle\partial \boldsymbol{u} / \partial t\rangle_{D}\right) .
$$

Compared to the K-J theorem, Eq. (8) is a general lift formula which contains only the two leading-order terms: the Lamb vector integral for the vortex force and the local vertical acceleration for the unsteady inertial effect. Particularly, in 2D, the sectional lift is given by

$$
L^{\prime}=\rho D\left(\left\langle u_{x} \omega_{y}\right\rangle_{D}-\left\langle\partial u_{z} / \partial t\right\rangle_{D}\right)
$$

where $\boldsymbol{u}=\left(u_{x}, u_{y}, u_{z}\right)$ is the fluid velocity vector, the $x$-, $y$ - and $z$-coordinates are in the freestream, spanwise and vertical directions, $D$ denotes the domain area in two dimensions, and $\langle\bullet\rangle_{D}$ becomes the area-averaged operator $\langle\bullet\rangle_{D}=D^{-1} \int_{D} \bullet d S$ in 2D. Rigorously speaking, the general lift formula, Eq. (8), is valid only for a rectangular control volume, which seems a considerably constrained case in theory. Nevertheless, this does not limit the usefulness of Eq. (8) because a simple rectangular domain is often used anyway for data processing in experiments and computations. Equation (9) is particularly suitable for lift estimation in experiments since the two velocity components $\left(u_{x}, u_{z}\right)$ on a streamwise cross-section and the spanwise vorticity $\omega_{y}$ are routinely measured using planar PIV in wind and water tunnels.

To compare Eq. (9) with the K-J theorem, the local effective velocity (the vorticity-weighted velocity) is defined as $u_{e f f}=u_{x} \omega_{y} /\left\langle\omega_{y}\right\rangle_{D}$, where $\left\langle\omega_{y}\right\rangle_{D}$ is the area-averaged vorticity. Further, by introducing the area-averaged effective velocity $U_{e f f}=\left\langle u_{e f f}\right\rangle_{D}$, Eq. (9) can be written in a form analogous to the K-J theorem

$$
L^{\prime}=\rho U_{e f f} \Gamma-\rho D\left\langle\partial u_{z} / \partial t\right\rangle_{D}
$$

where $\Gamma=\left\langle\omega_{y}\right\rangle_{D} D$ is the circulation. Unlike the classical K-J theorem $L_{K-J}^{\prime}=\rho U_{\infty} \Gamma$, the domain-averaged effective velocity $U_{e f f}$ rather than $U_{\infty}$ is used in Eq. (10), which depends on not only the velocity and vorticity distributions around a body but also time in general. In addition, the vertical acceleration term $\rho D\left\langle\partial u_{z} / \partial t\right\rangle_{D}$ explicitly represents the unsteady inertial effect that is omitted by the K-J theorem. For unsteady flows, application 
of the K-J theorem implies the quasi-steady-state assumption in which $L_{K-J}^{\prime}(t)$ is synchronized with $\Gamma(t)$. This assumption is generally problematic, which could lead to errors in the time-averaged magnitude and phase of the unsteady lift. To illustrate this point, by introducing a velocity difference $v(t)=U_{\text {eff }}(t)-U_{\infty}$ that represents a certain perturbation velocity, Eq. (10) gives $L^{\prime}(t)-L_{K-J}^{\prime}(t)=\rho v(t) \Gamma(t)-\rho D\left\langle\partial u_{z} / \partial t\right\rangle_{D}$. Clearly, it is indicated that $L^{\prime}(t)$ and $L_{K-J}^{\prime}(t)$ could considerably differ in the phase, amplitude and waveform of the unsteady lift particularly when the velocity fluctuation $v(t)$ and the acceleration term are large. The relation for the time-averaged lift is $\overline{L^{\prime}}-\bar{L}_{K-J}=\rho \overline{v \Gamma}-\rho D\left\langle\overline{\partial u_{z} / \partial t}\right\rangle_{D}$. The time-averaged magnitude change is given by the correlation term $\rho \overline{v(t) \Gamma(t)}$ plus the mean vertical acceleration term $\rho D\left\langle\overline{\partial u_{z} / \partial t}\right\rangle_{D}$, where the bar - denotes the time-average. If $\left\langle\partial u_{z} / \partial t\right\rangle_{D}$ is a symmetric function with respect to the time axis like a sinusoidal function, $\left\langle\overline{\partial u_{z} / \partial t}\right\rangle_{D}$ vanishes. In asymmetric wing flapping of a flyer, the mean acceleration term $\left\langle\overline{\partial u_{z} / \partial t}\right\rangle_{D}$ will contribute to the mean lift.

\section{B. The Kutta-Joukowski Theorem}

The classical K-J theorem is a reduced form of Eq. (10). In a steady $2 \mathrm{D}$ flow where $\left\langle\partial u_{z} / \partial t\right\rangle_{D}=0$, a vorticity patch bounded in a finite region that is much smaller than the rectangular control domain $D$ is considered. The $x$-component velocity can be decomposed into $u_{x}=U_{\infty}+u_{x}^{\prime}$, where $u_{x}^{\prime}$ is the velocity induced by the vorticity patch along the $x$-coordinate that is given by

$$
u_{x}^{\prime}(x, z)=\frac{1}{2 \pi} \iint_{D} \frac{(z-\eta) \omega_{y}(\xi, \eta)}{(x-\xi)^{2}+(z-\eta)^{2}} d \xi d \eta
$$

Therefore, since $u_{\text {eff }}=u_{x} \omega_{y} /\left\langle\omega_{y}\right\rangle_{D}=U_{\infty} \omega_{y} /\left\langle\omega_{y}\right\rangle_{D}+u_{x}^{\prime} \omega_{y} /\left\langle\omega_{y}\right\rangle_{D}$, the domain-averaged effective velocity is

$$
U_{e f f}=\left\langle u_{e f f}\right\rangle_{D}=U_{\infty}+\frac{1}{2 \pi} \frac{1}{\left\langle\omega_{y}\right\rangle_{D} D^{2}} \iiint \int \frac{(z-\eta) \omega_{y}(\xi, \eta) \omega_{y}(x, z)}{(x-\xi)^{2}+(z-\eta)^{2}} d \xi d \eta d x d z
$$

where the integration domain in both $(\xi, \eta)$ and $(x, z)$ is $D$. When the variables $(\xi, \eta)$ and $(x, z)$ are interchanged, the factor $z-\eta$ in Eq. (12) changes its sign. It is known that the integral in Eq. (12) must vanish such that $U_{e f f}=U_{\infty}$. In this case, the K-J theorem $L_{K-J}^{\prime}(t)=\rho U_{\infty} \Gamma$ is exactly recovered from Eq. (10). The above deduction is similar to that given by von Karman $\&$ Burgers ${ }^{23}$ for a bundle of vortex lines. The derivation of the K-J theorem from a general theory of viscous flows has been also given by $\mathrm{Wu}^{24}$, Wu et al. ${ }^{21}$ and $\mathrm{Schmitz} \&$ Chattot $^{25}$. In Sections 4 and 5, we will examine the above analysis based on direct numerical simulation (DNS) of low-Reynolds flows over a flat plate and a rectangular flat-plate wing.

\section{Numerical Method and Settings}

The 2D and 3D flows around a flat plate are simulated using an immersed boundary method based on discrete stream function formulation developed by Wang \& Zhang ${ }^{26}$. The incompressible Navier-Stokes equations with additional body forces and the continuity equation are used as the governing equations 


$$
\begin{gathered}
\frac{\partial \boldsymbol{u}}{\partial t}+\boldsymbol{u} \cdot \nabla \boldsymbol{u}=-\nabla p+\frac{1}{\operatorname{Re}_{c}} \nabla^{2} \boldsymbol{u}+\boldsymbol{f}, \\
\nabla \cdot \boldsymbol{u}=0,
\end{gathered}
$$

where, $\boldsymbol{u}$ is the non-dimensional velocity, $p$ the non-dimensional pressure, and $\boldsymbol{f}$ the non-dimensional body forces representing the effects of the boundaries. The Reynolds number is defined as $\operatorname{Re}_{c}=U c / v$, where $U$ is the upstream flow velocity, $c$ the chord length, and $v$ the kinematic viscosity. Eqs. (13) and (14) are solved on a unstructured Cartesian (Eulerian) mesh in the framework of discrete stream function formulation. The plate is marked using a set of Lagrangian points. The Eulerian mesh and Lagrangian points are coupled using the interpolation based on discrete $\delta$ function. The discrete form of body force $f$ is determined implicitly by solving a linear system to implement the non-slip boundary conditions on the surface of the plate.

The simulations are conducted in a domain of $[-12,20] \times[-16,16]$ in streamwise $(x)$ and vertical $(z)$ directions for $2 \mathrm{D}$ flows, and $[-12,20] \times[-16,16] \times[-16,16]$ in streamwise $(x)$, spanwise $(y)$ and vertical $(z)$ directions for 3D flows. The center of the plate is positioned at the origin. The unstructured mesh with hanging-node is used in the simulations to refine the mesh around the plate. The minimum grid size used for the $2 \mathrm{D}$ and $3 \mathrm{D}$ flows are $0.01 \mathrm{c}$ and $0.02 \mathrm{c}$, respectively. The time step is selected to keep the maximum CFL number at 0.5 in the simulations. The maximum CFL number is defined as

$$
C F L_{\text {max }}=\max \left(u_{f i} d t\left(\frac{1}{d_{1}}+\frac{1}{d_{2}}\right)\right), \quad i=1,2,3, \cdots, \text { number of face }
$$

where $u_{f i}$ is the normal component of the velocity at face $i$, and $d_{l}$ and $d_{2}$ the distances between the centroid of face $i$ and the centroids of its two neighboring cells, respectively. The uniform flow $(U, 0,0)$ is specified at the inlet, the free convection boundary condition is used at the outlet. The non-slip boundary condition is set at the surface of the plate. The symmetric boundary conditions are used at the other boundaries. The flow is uniform $(U, 0,0)$ at $t=0$ before the plate instantaneously appears at $t=0^{+}$.

To validate the present numerical method by comparing with the published results by Taira $\&$ Colonius ${ }^{27}$, flows around an impulsively-starting rectangular flat-plate wing with the aspect ratio $A R=2$ are simulated at different angles of attack (AoA, $\alpha$ ) for the Reynolds number based on the chord $R e_{c}=100$. The lift and drag coefficients of the flat-plate wing calculated as a function of AoA are in good agreement with the numerical and experimental results obtained by Taira \& Colonius ${ }^{27}$. More details about the numerical method and its validations are given in our previous work ${ }^{26}$.

\section{Flat Plate}

\section{A. Stationary Plate}

The sectional lift of a stationary flat plate (a flat-plate airfoil) is calculated by using the K-J theorem $L_{K-J}^{\prime}=\rho U_{\infty} \Gamma$ and the general lift formula $L^{\prime}=\rho U_{e f f} \Gamma-\rho D\left\langle\partial u_{z} / \partial t\right\rangle_{D}$. The domain $D$ for the circulation integral and the general lift formula is a sufficiently large area on the cross-section plane $(x, z)$. Figure 2 shows typical rectangular domains used here. The left boundary is located at $x / c=-1$ upstream the leading edge, the right boundary at the trailing edge and two locations in the wake, and the top and bottom boundaries at $z / c= \pm 6$, where $c$ is the chord. The main results in this paper are calculated based on the domain $D_{1}$ where the right boundary is at the trailing edge. For a stationary flat plate in the incoming freestream flow that starts suddenly, as shown in Fig. 3, the time-averaged lift coefficients calculated by using the K-J theorem and the general lift formula as a function of AoA are directly compared with those obtained from Eq. (1) for $R e_{c}=300$. The timeaveraged lift coefficient is defined as $\overline{C_{l}}=\overline{L^{\prime}} /\left(\rho U_{\infty}^{2} c / 2\right)$, where the bar - denotes the time-average. In the relevant figures in this paper, "DNS" denotes the result calculated directly from Eq. (1), "K-J" denotes the K-J theorem, "General" denotes the general lift formula [Eq. (8) or (9)], and "Thin Wing" denotes the thin-wing theory. 
The K-J theorem predicts the time-averaged lift coefficients $\overline{C_{l}}$ well for $\alpha \leq 30^{\circ}$, but it over-predicts $\overline{C_{l}}$ in $30^{\circ}<\alpha \leq 50^{\circ}$ and considerably under-predicts $\bar{C}_{l}$ for $\alpha>50^{\circ}$. The prediction by the classical thin-wing theory $\overline{C_{l}}=2 \pi \alpha$ is also plotted in Fig. 3 as a reference. In contrast, the general lift formula gives $\overline{C_{l}}$ that is in very good agreement with DNS. Table 1 lists the time-averaged lift and drag coefficients of the flat plate at different AoAs along with the vortex shedding Strouhal numbers. The error in $\overline{C_{l}}$ given by the K-J theorem is less than $7 \%$ for $\alpha \leq 30^{\circ}$, but it increases to about $20 \%$ in $30^{\circ}<\alpha \leq 50^{\circ}$. The error in $\overline{C_{l}}$ given by the general lift formula is less than $2 \%$ for $10^{\circ}<\alpha \leq 80^{\circ}$.

Figure 4 shows the snap-shot fields of the non-dimensional vorticity, vertically-projected Lamb vector and vertically-projected acceleration around a flat plate at the non-dimensional time $t^{*}=147.6$ for $\operatorname{Re}_{c}=$ 300 and $\alpha=30^{\circ}$. Throughout this paper, the non-dimensional vorticity, vertically-projected Lamb vector, vertically-projected acceleration and time are defined as $\boldsymbol{\omega}\left(c / U_{\infty}\right), \boldsymbol{k} \cdot(\boldsymbol{u} \times \boldsymbol{\omega})\left(c / U_{\infty}^{2}\right)$, $\boldsymbol{k} \cdot \partial \boldsymbol{u} / \partial t\left(c / U_{\infty}^{2}\right)$, and $t^{*}=t\left(U_{\infty} / c\right)$, respectively. According to Eq. (8), the vertically-projected Lamb vector and vertically-projected acceleration are the leading terms contributing to the lift. Vortex shedding occurs when $\alpha \geq 20^{\circ}$ even though the flat plate is stationary, and therefore $C_{l}$ is time-dependent. The vortex-shedding Strouhal number based on the front-projected height, defined as $S t=f_{s} c \sin \alpha / U_{\infty}$, is $S t=0.14-0.17$ as shown in Table 1 , which is consistent with the experimental data

Table 1. Vortex shedding Strouhal number, time-averaged lift and drag coefficients of the stationary 2D flat plate

\begin{tabular}{|c|c|c|c|c|c|c|c|}
\hline$\alpha$ & $S t$ & $\bar{C}_{d}$ & $\bar{C}_{l}$ & $\bar{C}_{l, g e n}$ & Error of $\bar{C}_{l, g e n}$ & $\bar{C}_{l, K-J}$ & Error of $\bar{C}_{l, K-J}$ \\
\hline 0 & -- & 0.2 & 0.0 & 0.0 & -- & 0.0 & -- \\
\hline 10 & -- & 0.25 & 0.68 & 0.67 & $1 \%$ & 0.69 & $1 \%$ \\
\hline 20 & 0.16 & 0.45 & 0.92 & 0.92 & 0 & 0.92 & 0 \\
\hline 30 & 0.17 & 0.83 & 1.22 & 1.22 & 0 & 1.31 & $7 \%$ \\
\hline 40 & 0.17 & 1.30 & 1.36 & 1.39 & $2 \%$ & 1.58 & $16 \%$ \\
\hline 50 & 0.15 & 1.88 & 1.39 & 1.41 & $1 \%$ & 1.47 & $6 \%$ \\
\hline 60 & 0.15 & 2.62 & 1.40 & 1.41 & $1 \%$ & 1.19 & $15 \%$ \\
\hline 70 & 0.14 & 3.02 & 1.03 & 1.02 & $1 \%$ & 0.85 & $17 \%$ \\
\hline 80 & 0.14 & 3.23 & 0.53 & 0.52 & $2 \%$ & 0.26 & $50 \%$ \\
\hline 90 & 0.14 & 3.34 & 0.0 & 0.0 & -- & 0.0 & -- \\
\hline
\end{tabular}

Note: $\bar{C}_{l}$ and $\bar{C}_{d}$ denote the time-averaged lift and drag coefficients directly obtained from DNS for $R e_{c}=300$, and $\bar{C}_{l, K-J}$ and $\bar{C}_{l, g e n}$ denote the time-averaged lift coefficients given by using the K-J theorem and the general lift formula, respectively.

for flat-plates ${ }^{28}$. Figure 5(a) shows the time histories of the lift coefficient of the stationary plate for $R e_{c}=300$ and $\alpha=30^{\circ}$. The general lift formula gives $C_{l}$ that is in good agreement with DNS in both the amplitude and phase.

8

American Institute of Aeronautics and Astronautics 
In this case, as shown in Fig. 5(b), the Lamb vector integral is the major contribution to the lift, while the acceleration term has a relatively small effect. As indicated in Fig. 4(c), the verticallyprojected acceleration around the flat plate is indeed small.

Even for the stationary plate, as indicated in Fig. 5(a), there is a large phase difference between $L^{\prime}(t)$ given by the general lift formula and $L_{K-J}^{\prime}(t)$ by the K-J theorem. The amplitude given by the K-J theorem is lower than that given by the general lift formula particularly when the flow becomes unsteady for large AoAs. The K-J theorem $L_{K-J}^{\prime}(t)=\rho U_{\infty} \Gamma(t)$ indicates that the lift on the flat plate has the same phase with the circulation around the wing since $U_{\infty}$ is a constant for a stationary flat plate. However, the general lift formula $L^{\prime}=\rho U_{\text {eff }} \Gamma-\rho D\left\langle\partial u_{z} / \partial t\right\rangle_{D}$ shows that the lift is out of phase with the circulation due to not only the time-dependent effective velocity $U_{\text {eff }}(t)$ but also the unsteady acceleration term $\left\langle\partial u_{z} / \partial t\right\rangle_{D}$. In this case, $L^{\prime}(t)$ is out of phase with $\Gamma(t)$ by about $180^{\circ}$, and the phase of $L^{\prime}(t)$ is dominated by the phase of $U_{\text {eff }}(t)$. The K-J theorem as a quasi-steady model does not reflect the unsteady coupling between $U_{\text {eff }}(t)$ and $\Gamma(t)$. The time-averaged lift difference given by the generalized lift formula and the K-J theorem is

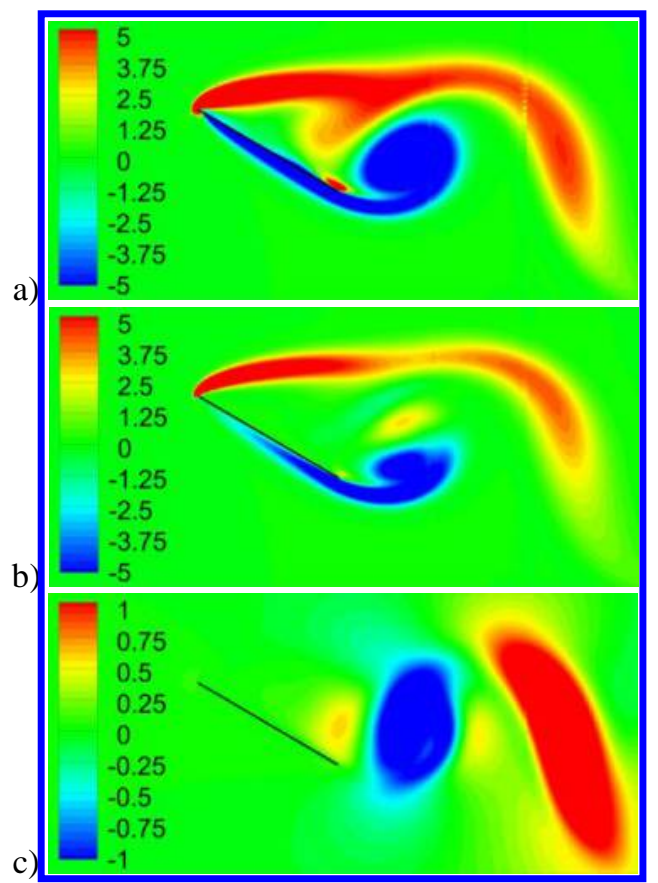

Figure 4. Non-dimensional snap-shot fields around the stationary flat plate for $\operatorname{Rec}=\mathbf{3 0 0}$ and $\alpha=30^{\circ}$, (a) vorticity, (b) verticallyprojected Lamb vector, and (c) verticallyprojected acceleration $\overline{L^{\prime}}-\bar{L}_{K-J}^{\prime}=\rho C_{v \Gamma}|\bar{v}||\bar{\Gamma}|-\rho D\left\langle\overline{\partial u_{z} / \partial t}\right\rangle_{D}$, where a correlation coefficient $C_{v \Gamma}=\overline{v \Gamma} /|\bar{v}||\bar{\Gamma}|$ is introduced. This difference is proportional to $\bar{v}=U_{\infty}\left[\overline{U_{\text {eff }}} / U_{\infty}-1\right]$. It is found that $\overline{U_{\text {eff }}} / U_{\infty}$ increasingly deviates from one as AoA increases after $\alpha>30^{\circ}$, which corresponds to the difference $\overline{L^{\prime}}-{\overline{L^{\prime}}}_{K-J}$ shown in Fig. 3.
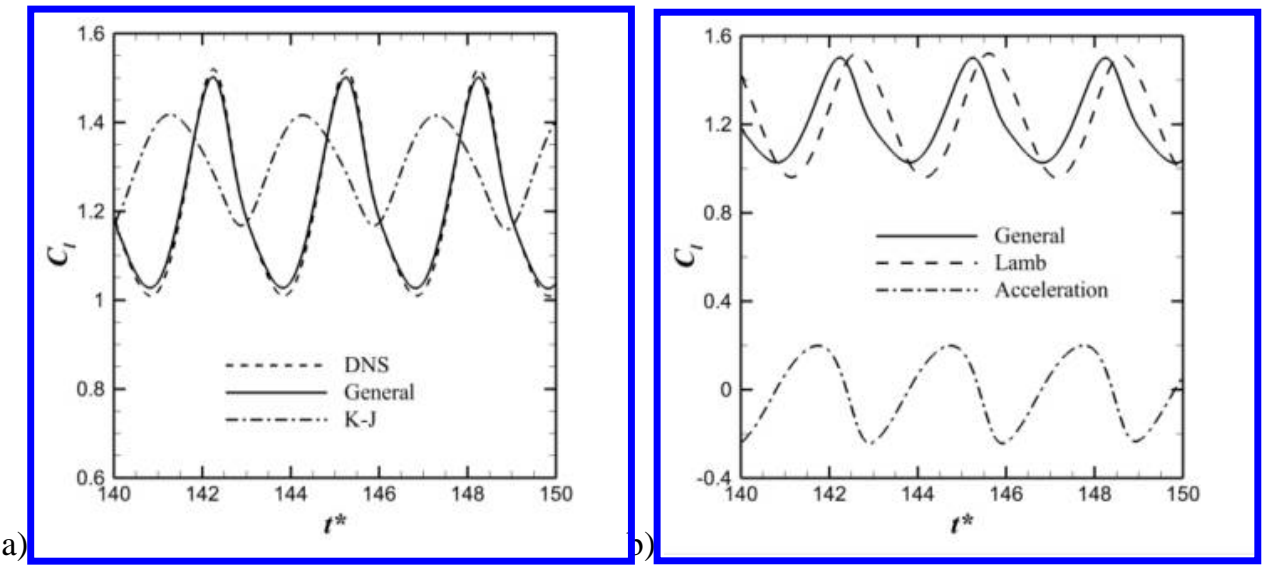

Figure 5. Time histories of the lift coefficient of the stationary flat plate for $\boldsymbol{R e}_{c}=\mathbf{3 0 0}$ and $\alpha=30^{\circ}$, (a) comparison between DNS, the K-J theorem and the general lift formula, and (b) contributions from the Lamb vector integral and acceleration term

In the derivation of the general lift formula Eq. (8), the rectangular domain is not specified. Theoretically speaking, lift calculation is independent of a rectangular domain selected, but actual result is affected by the selection of a domain depending on the numerical or measurement accuracy. To illustrate this issue, three 
rectangular domains shown in Fig. 2 are used for Eq. (8). Figure 6 shows the time histories of the lift coefficient of the stationary flat plate calculated based on the three domains for $R e_{c}=300$ and $\alpha=30^{\circ}$. The value of $C_{l}$ calculated based on the domain $D_{l}$ where the right boundary is at the trailing edge is very close to DNS. In contrast, calculations based on the domains $D_{2}$ and $D_{3}$ that contain the portion of the wake structures under-predict $C_{l}$ somewhat particularly near the peaks. The more pronounced deviation is found for the larger domain $D_{3}$. The underestimate is mainly due to the coarser grid in the wake in computations such that the vorticity field is not fully resolved there. Therefore, the main results in this paper are obtained based on the domain $D_{1}$.

\section{B. Flapping Plate}

The applicability of the K-J theorem and the general lift formula to a flapping plate is examined. The kinematics of a flapping flat plate is prescribed as a superposition of the pitching and heaving motions by

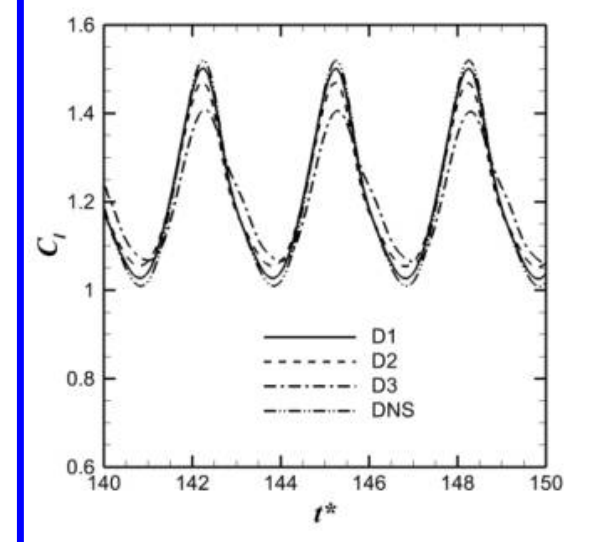

Figure 6. Time histories of the lift coefficient of the stationary plate calculated based on three domains for $\boldsymbol{R e}_{c}=\mathbf{3 0 0}$ and $\alpha=30^{\circ}$

$$
\alpha=\alpha_{0}+\alpha_{m} \cos (2 \pi f t), z_{c}=A \sin (2 \pi f t),
$$

where the time-averaged AoA is $\alpha_{0}=10^{\circ}$, the pitching amplitude is $\alpha_{m}=30^{\circ}$, the heaving amplitude is $A=c / 4$, $z_{c}$ is the vertical position of the plate center and $f$ is the flapping frequency. The flapping Strouhal number is $S t_{f}=2 \mathrm{Af} / U_{\infty}=0.3$ that is close the optimal Strouhal number $\left(0.2<S t_{f}<0.4\right)$ of various flying and swimming animals for high power efficiency ${ }^{29}$. The corresponding reduced frequency is $f c / U_{\infty}=0.6$ The total sectional force on the plate calculated by using the K-J theorem is $F^{\prime}=\rho U \Gamma$, where $U=\sqrt{U_{\infty}^{2}+U_{c}^{2}}$ is the effective local upstream velocity, $U_{\infty}$ is the freestream velocity, $U_{c}=\dot{y}_{c}=2 \pi f A \cos (2 \pi f t)$ is the heaving velocity of the plate center. The direction of the effective upstream velocity is given by $\theta=\arctan \left(-U_{c} / U_{\infty}\right)$. The sectional lift is the vertical component of the total force $L^{\prime}=F^{\prime} \cos (\theta)$.

Figure 7 shows the snap-shot fields of the non-dimensional vorticity, vertically-projected Lamb vector, and verticallyprojected acceleration around the flapping flat plate at one moment for $R e_{c}=300$. Compared with Fig. 4 for the stationary plate, the significant vertically-projected acceleration of fluid is found around the flapping plate, which contributes to the unsteady lift. Figure 8 shows the histories of the lift coefficient of the flapping flat plate. The general lift formula gives the result that is consistent with DNS. The Lamb vector integral itself has a considerable phase shift compared to DNS, while the acceleration term has a significant contribution to the unsteady lift in the phase, amplitude and waveform. Nevertheless, the sum of the Lamb vector integral and the acceleration term recovers the true waveform.

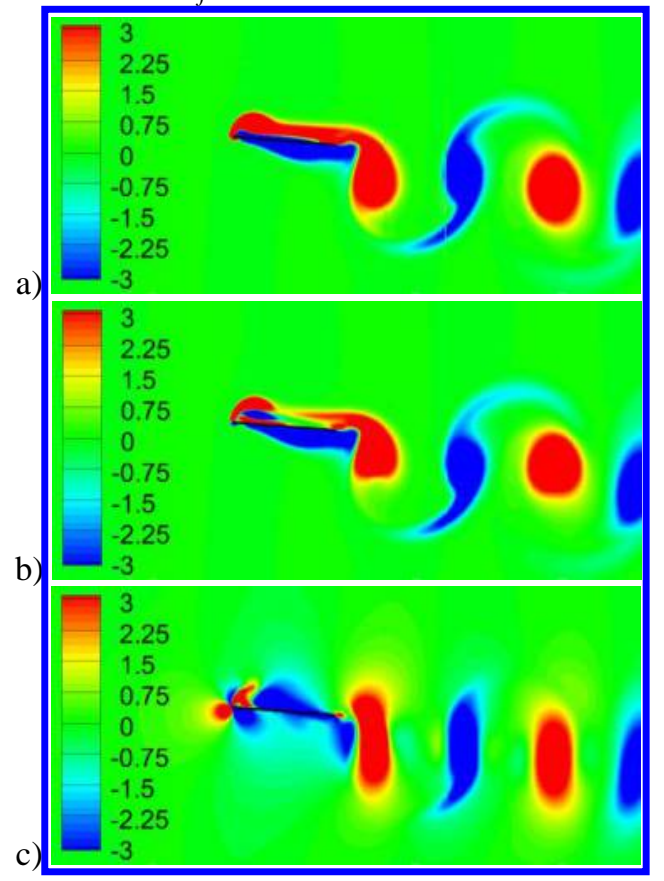

Figure 7. Non-dimensional snap-shot fields around the flapping flat plate at one moment for $\boldsymbol{R e}_{c}=300$, (a) vorticity, (b) verticallyprojected Lamb vector, and (c) verticallyprojected acceleration 


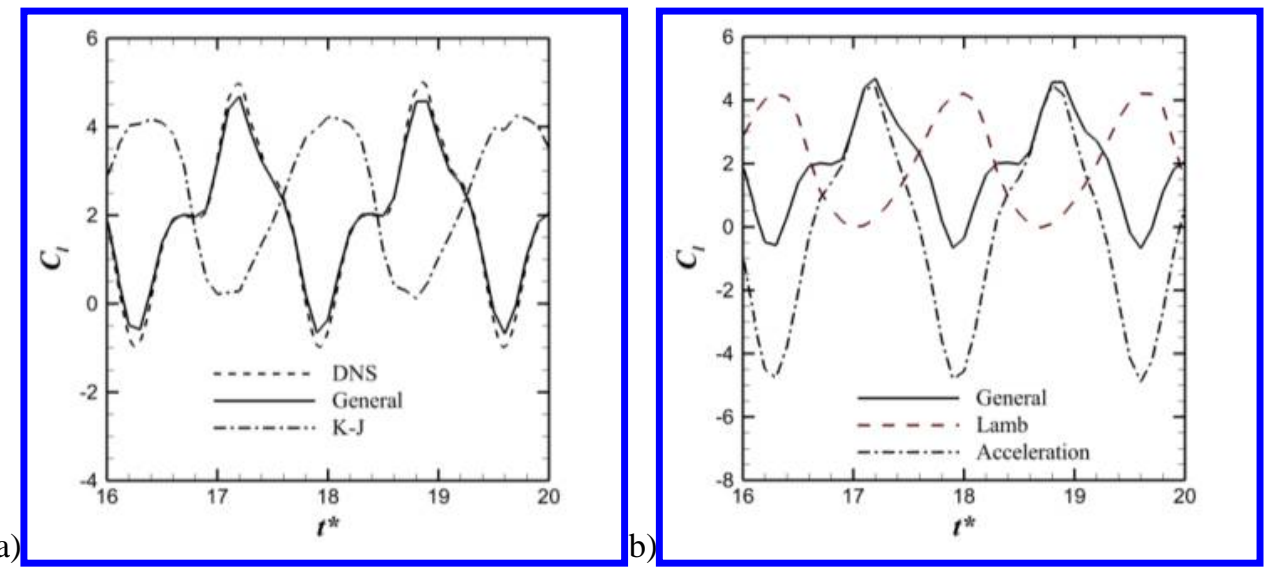

Figure 8. Histories of the lift coefficient of the flapping flat plate for $R e_{c}=300$, (a) comparison between DNS, the K-J theorem and the general lift formula, and (b) contributions from the Lamb vector integral and acceleration term

The time-averaged lift coefficients calculated based on different methods are showed in Table 2 along with the contributions of the Lamb vector integral and acceleration term to the time-averaged lift. It is shown that the timeaveraged lift calculated by using the K-J theorem just captures the contribution of Lamb vector integral. In this case, the flapping kinematics of the plate is not symmetric with respect to the time axis, the time-averaged quantity $\left\langle\overline{\partial u_{z} / \partial t}\right\rangle_{D}$ contributes about $10 \%$ of the time-averaged lift. The more asymmetric flapping of a bird or bat may contribute more to the mean lift through the acceleration term. When the time-averaged AoA in Eq. (16) is $\alpha_{0}=0^{\circ}$ in the symmetric flapping, the time-averaged contribution of the acceleration term is zero. The above analysis indicates that a quasi-steady model like the K-J theorem (even the Lamb vector integral alone) based on the snap-shot vorticity fields cannot capture the unsteady nature of the lift in flapping flight. The acceleration term for the unsteady inertial effect is significant, and time-resolved velocity measurements or computations are required to obtain the unsteady lift.

Table 2. Time-averaged lift coefficient of the flapping flat plate

\begin{tabular}{|l|l|l|l|l|}
\hline $\bar{C}_{l, D N S}$ & $\bar{C}_{l, \text { gen }}$ & $\bar{C}_{l, K-J}$ & $\bar{C}_{l, \text { Lamb }}$ & $\bar{C}_{l, a c c}$ \\
\hline 2.07 & 2.07 & 1.86 & 1.86 & 0.21 \\
\hline
\end{tabular}

Note: $\bar{C}_{l, D N S}$ represents the time-averaged lift coefficient calculated directly from DNS for $R e_{c}=300$ and $S t_{f}=0.3, \bar{C}_{l, g e n}$ and $\bar{C}_{l, K-J}$ are given by the general lift formula and the K-J theorem, respectively, and $\bar{C}_{l, \text { Lamb }}$ and $\bar{C}_{l, a c c}$ represent the contributions of the Lamb vector integral and acceleration term in the general lift formula, respectively.

\section{Rectangular Flat-Plate Wing}

\section{A. Stationary Wing}

Flow fields over a stationary rectangular flat-plate wing with $A R=4$ are calculated at different AoAs for $R e_{c}=$ 300 after the incoming freestream flow starts suddenly. For a finite wing, Eq. (8) can be expressed as

$$
L=\rho V_{D}\left(\left\langle u_{x} \omega_{y}\right\rangle_{D}-\left\langle u_{y} \omega_{x}\right\rangle_{D}\right)-\rho V_{D}\left\langle\partial u_{z} / \partial t\right\rangle_{D} .
$$

The first term in the Lamb vector integral on RHS of Eq. (17) is the dominant component, and the second term is a relatively small component for a rectangular wing since interaction between the spanwise velocity and the streamwise vorticity is weak. The third term is the acceleration term. Figure 9 shows the time-averaged lift 
coefficient as a function of AoA. The general lift formula predicts well the lift for all AoAs. In planar PIV measurements, 2D velocity fields on different spanwise slices along the wing span are usually obtained, and therefore the sectional lift $L^{\prime}$ is calculated for each slice. Then, the total lift $L$ of the wing is obtained by summing the contributions from

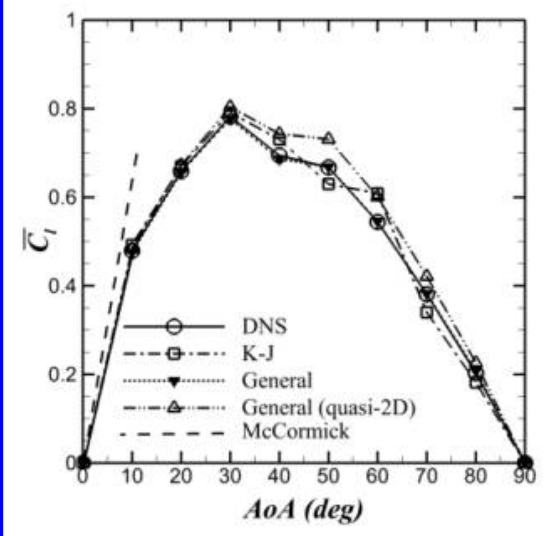

Figure 9. The time-averaged lift coefficient as a function of AoA for the stationary rectangular flat-plate wing with $A R=4$ for $\operatorname{Re}=300$ all the slices. This approach is a quasi-2D approximation where only the first term in the Lamb vector integral in Eq. (17) is used since the streamwise vorticity and the spanwise velocity cannot be measured at the same time in planar PIV. The general lift formula with the quasi-2D approximation is compared with DNS in Fig. 9. It is indicated that this quasi-2D method is reasonably good even though the time-averaged lift is over-predicted slightly for $\alpha>30^{\circ}$. Interestingly, in this case, the K-J theorem gives the time-averaged lift that is consistent with DNS as well. This may be coincident. For comparison, Fig. 9 also includes the lift for a finite wing given by McCormick's formula where the lift slope is ${ }^{30}$

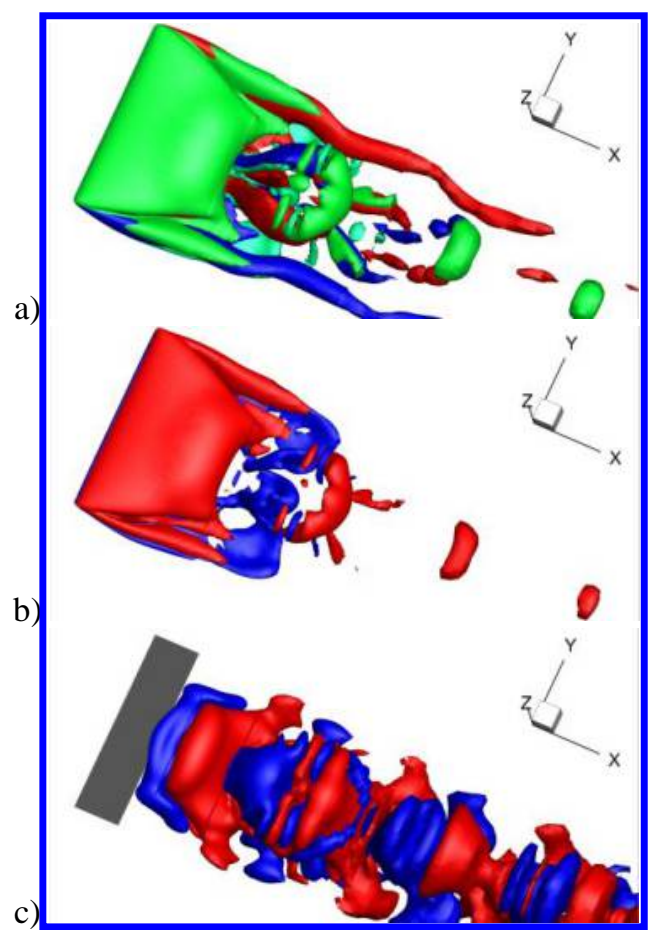

Figure 10. Non-dimensional snap-shot fields around a stationary rectangular flat-plate wing with $A R=4$ for $R e_{c}=300$ and $\alpha=30^{\circ}$, (a) vorticity, (b) verticallyprojected Lamb vector, and (c) verticallyprojected acceleration

$$
a=\frac{d C_{l}}{d \alpha}=\frac{a_{0} A R}{A R+2(A R+4) /(A R+2)},
$$

where $a_{0}=2 \pi$ according to the thin-wing theory.
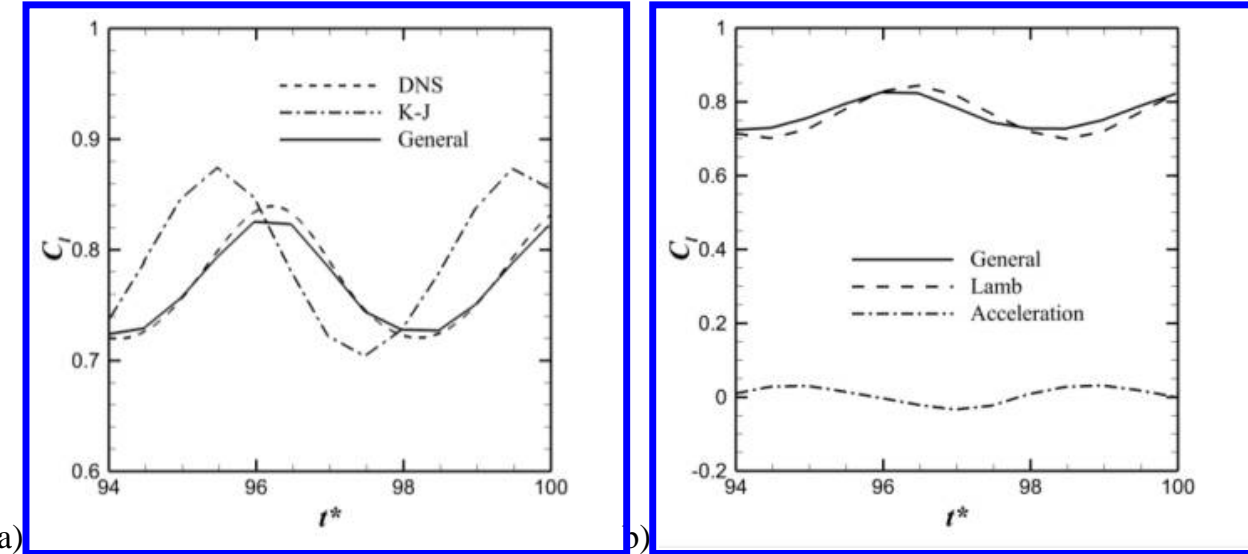

Figure 11. Histories of the lift coefficient of the stationary rectangular flat-plate wing with $A R=4$ for $\boldsymbol{R} \boldsymbol{e}_{c}=300$ and $\alpha=30^{\circ}$, (a) comparison between DNS, the K-J theorem and the general lift formula, and (b) contributions from the Lamb vector integral and acceleration term 
Figure 10 shows the snap-shot fields of the non-dimensional vorticity, vertically-projected Lamb vector, and verticallyprojected acceleration around a stationary rectangular flat-plate wing with $A R=4$ at one moment for $R e_{c}=300$ and $\alpha=30^{\circ}$. Similar to Fig. 4 for the $2 \mathrm{D}$ case, the vertically-projected acceleration of fluid around the wing is small, indicating its contribution to the time-averaged lift is not significant. Figure 11(a) shows the histories of the lift coefficient of the wing for $R e_{c}=300$ and $\alpha=30^{\circ}$. The general lift formula gives a consistent result with DNS. In contrast, the lift coefficient given by the K-J theorem has a lower time-averaged value and a significant phase shift. It is indicated that the first term the Lamb vector integral in Eq. (17) makes the most contribution to the lift from the spanwise vorticity field. Although the contribution of the acceleration to the time-averaged lift is relatively small for the stationary wing, it affects the phase as indicated in Fig. 11(b).

\section{B. Flapping Wing}

The kinematics of a flapping rectangular flat-plate wing is prescribed using Eq. (16). Figure 12 shows the snap-shot fields of the non-dimensional vorticity, vertically-projected Lamb vector, and vertically-projected acceleration around the flapping wing at one moment for $R e_{c}=300$. In contrast to the stationary wing shown in Fig. 10, the vertically-projected acceleration around the flapping wing is significantly large. Figure 13(a) shows the histories of the lift coefficient of the flapping wing. The general lift formula gives the consistent result with DNS. The lift predicted by the K-J theorem has not only a considerable phase shift but also a different waveform compared to DNS. Interestingly, in this case, the Lamb vector integral in the general lift formula gives a waveform that is close to that given by the K-J theorem. Nevertheless, as indicated in Fig. 13(b), the acceleration term for the unsteady inertial effect has a considerably large effect on both the amplitude and phase of the lift for the flapping wing. The sum of the Lamb vector integral and the acceleration term recovers the true waveform. Table 3 gives the time-averaged lift coefficients calculated based on different methods along with the contributions of the Lamb vector term and acceleration term. Similar to the 2D flow case in Table 2,
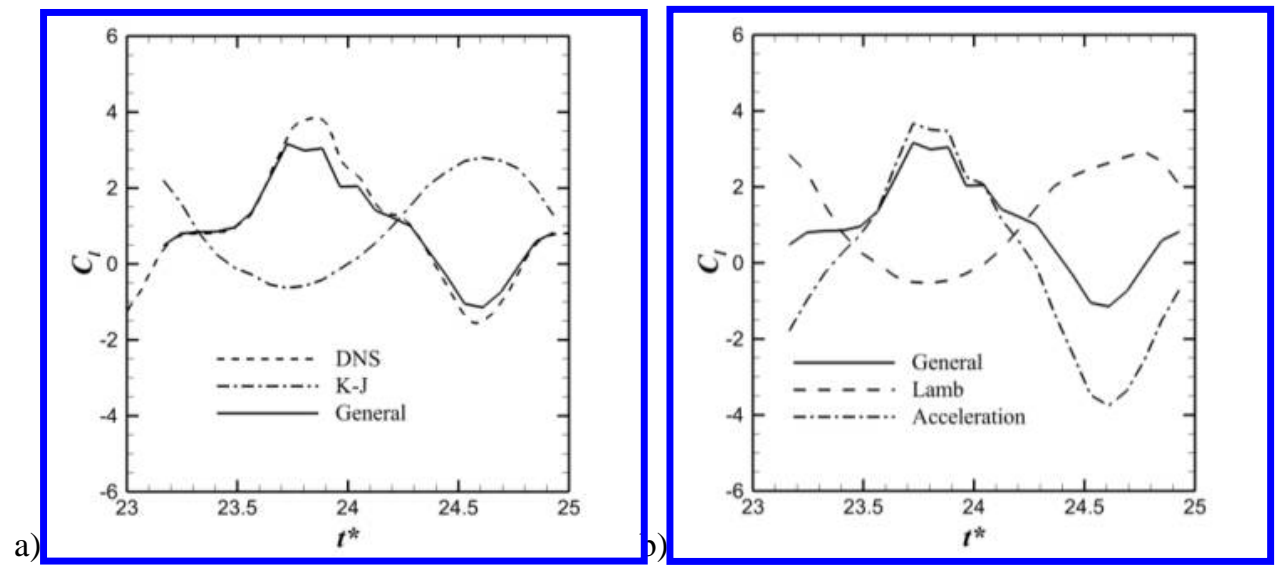

Figure 13. Histories of the lift coefficient for the flapping rectangular flat-plate wing with $A R=4$ for $R e_{c}=$ 300, (a) comparison between DNS, the K-J theorem and the general lift formula, and (b) contributions from the Lamb vector integral and acceleration term. 
the K-J theorem does not capture the contribution of the flow acceleration to the time-averaged lift. The acceleration term $\left\langle\overline{\partial u_{z} / \partial t}\right\rangle_{D}$ contributes about $10 \%$ of the time-averaged lift.

Table 3. Time-averaged lift coefficient of the flapping rectangular flat-plate wing

\begin{tabular}{|l|l|l|l|l|}
\hline $\bar{C}_{l, \mathrm{DNS}}$ & $\bar{C}_{l, \mathrm{gen}}$ & $\bar{C}_{l, \mathrm{~K}-\mathrm{J}}$ & $\bar{C}_{l, \text { Lamb }}$ & $\bar{C}_{l, a c c}$ \\
\hline 1.13 & 1.12 & 1.01 & 1.02 & 0.10 \\
\hline
\end{tabular}

Note: The notations are defined in Table 2.

\section{Conclusions}

The general lift formula is given for a rectangular control volume in the framework of the general viscous flow theory, and it has a lucid form with the two leading-order terms: the Lamb vector integral for the vortex force and the acceleration term of fluid for the unsteady inertial effect. Since the Kutta-Joukowski (K-J) theorem is just a reduced case from this formula, the limitations of the K-J theorem can be critically examined. It is found that the application of the K-J theorem to unsteady low-Reynolds-number flows will inevitably lead to errors in the phase, amplitude and waveform of the unsteady lift particularly on a flapping wing. From a physical point of view, the K-J theorem as a quasi-steady model ignores interaction or correlation between the domain-averaged effective velocity and the circulation in unsteady flows. Furthermore, the K-J theorem does not take into account the acceleration of fluid that becomes significant around flapping wings as the unsteady inertial effect on the lift. The general lift formula is validated and the relevant theoretical arguments are supported by direct numerical simulation (DNS) on the stationary and flapping flat plates and rectangular wings. As indicated by DNS, the fields of the verticallyprojected Lamb vector and vertically-projected acceleration around a flapping wing, rather than the vorticity field itself, are more directly responsible to the lift generation. Due to its simplicity, the general lift formula is particularly useful for estimation of the unsteady lift from velocity fields obtained in measurements and computations.

\section{Acknowledgments}

This work was supported by Chinese Academy of Sciences under the Innovative Projects (KJCX-SW-L08) and (KJCX3-SYW-S01); National Natural Science Foundation of China under Project Nos.10325211, 10628206, 10732090 and 10872201. Tianshu Liu would like to acknowledge the hospitality received at LNM during his visit where he accomplished this work. The authors also like to thank the National Supercomputing Center in Tianjin (NSCC-TJ) for the allocation of computing time.

\section{References}

$>^{1}$ Platzer, M. F., Jones, K. D., Young, J., and S. Lai, J. C. "Flapping Wing Aerodynamics: Progress and Challenges," AIAA Journal Vol. 46, No. 9, 2008, pp. 2136-2149.

${ }^{2}$ Shyy, W., Lian, Y., Tang, J., Viieru, D., and Liu, H. Aerodynamics of low Reynolds number flyers. Cambridge: Cambridge University Press, 2008.

${ }^{3}$ Spedding, G. R., Rosen, M., and Hedenstrom, A. "A family of vortex wakes generated by a thrush nightingale in free flight in a wind tunnel over its entire natural range of flight speeds," Journal Of Experimental Biology Vol. 206, No. 14, 2003, pp. 2313-2344.

${ }^{4}$ Hedenstrom, A., Johansson, L. C., Wolf, M., von Busse, R., Winter, Y., and Spedding, G. R. "Bat flight generates complex aerodynamic tracks," Science Vol. 316, No. 5826, 2007, pp. 894-7.

${ }^{5}$ Muijres, F. T., Johansson, L. C., Barfield, R., Wolf, M., Spedding, G. R., and Hedenstrom, A. "Leading-edge vortex improves lift in slow-flying bats," Science Vol. 319, No. 5867, 2008, pp. 1250-3.

${ }^{6}$ Hubel, T. Y., Hristov, N. I., Swartz, S. M., and Breuer, K. S. "Time-resolved wake structure and kinematics of bat flight," Experiments in Fluids Vol. 46, No. 5, 2009, pp. 933-943.

${ }^{7}$ Hubel, T. Y., Riskin, D. K., Swartz, S. M., and Breuer, K. S. "Wake structure and wing kinematics: the flight of the lesser dog-faced fruit bat, Cynopterus brachyotis," Journal of Experimental Biology Vol. 213, No. 20, 2010, pp. 3427-3440.

${ }^{8}$ Rayner, J. M. V. "Vortex theory of animal flight 1. vortex wake of a hovering animal," Journal of Fluid Mechanics Vol. 91, No. APR, 1979, pp. 697-730. 
${ }^{9}$ Rayner, J. M. V. "Vortex theory of animal flight 2. forward flight of birds," Journal Of Fluid Mechanics Vol. 91, No. APR, 1979, pp. 731-763.

${ }^{10}$ Ellington, C. P. "The Aerodynamics of Hovering Insect Flight. V. A Vortex Theory," Philosophical Transactions of the Royal Society B: Biological Sciences Vol. 305, No. 1122, 1984, pp. 115-144.

${ }^{11}$ Watts, P., Mitchell, E. J., and Swartz, S. M. "A computational model for estimating the mechanics of horizontal flapping flight in bats: Model description and validation," Journal of Experimental Biology Vol. 204, No. 16, 2001, pp. 2873-2898.

${ }^{12}$ Hedrick, T. L., Tobalske, B. W., and Biewener, A. A. "Estimates of circulation and gait change based on a threedimensional kinematic analysis of flight in cockatiels (Nymphicus hollandicus) and ringed turtle-doves (Streptopelia risotia)," Journal Of Experimental Biology Vol. 205, No. 10, 2002, pp. 1389-1409.

${ }^{13}$ Sane, S. P. "The aerodynamics of insect flight," Journal of Experimental Biology Vol. 206, No. 23, 2003, pp. 4191-4208.

${ }^{14}$ Ansari, S. A., Zbikowski, R., and Knowles, K. "Aerodynamic modelling of insect-like flapping flight for micro air vehicles," Progress In Aerospace Sciences Vol. 42, No. 2, 2006, pp. 129-172.

${ }^{15}$ Milne-Thomson, L. M. Theoretical hydrodynamics: Dover Publications, 1996.

${ }^{16}$ Anderson, J. D. Fundamentals of Aerodynamics: McGraw-Hill, 2010.

${ }^{17}$ Liu, T., and Sullivan, J. P. Pressure and temperature sensitive paints: Springer, 2004.

${ }^{18}$ Liu, T., Montefort, J., Woodiga, S., Merati, P., and Shen, L. "Global Luminescent Oil-Film Skin-Friction Meter," AIAA Journal Vol. 46, No. 2, 2008, pp. 476-485.

${ }^{19} \mathrm{Wu}$, J. Z., and Wu, J. M. "Vorticity dynamics on boundaries," Advances in Applied Mechanics Vol. 32, 1996, pp. 119-275.

${ }^{20}$ Noca, F., Shiels, D., and Jeon, D. "A comparison of methods for evaluating time-dependent fluid dynamic forces on bodies, using only velocity fields and their derivatives," Journal Of Fluids And Structures Vol. 13, No. 5, 1999, pp. 551-578.

${ }^{21} \mathrm{Wu}$, J. Z., Ma, H. Y., and Zhou, M. D. Vorticity and vortex dynamics: Springer, 2006.

${ }^{22} \mathrm{Wu}$, J. Z., Lu, X. Y., and Zhuang, L. X. "Integral force acting on a body due to local flow structures," Journal Of Fluid Mechanics Vol. 576, 2007, pp. 265-286.

${ }^{23}$ von Karman, T., and Burgers, J. "General aerodynamic theory -- perfect fluids," Aerodynamic Theory. Dover publications, 1963, 1935.

$\vee^{24} \mathrm{Wu}$, J. C. "Theory for aerodynamic force and moment in viscous flows," Aiaa Journal Vol. 19, No. 4, 1981, pp. $432-441$.

${ }^{25}$ Schmitz, S., and Chattot, J.-J. "Flow physics and Stokes' theorem in wind turbine aerodynamics," Computers \& Fluids Vol. 36, No. 10, 2007, pp. 1583-1587.

${ }^{26}$ Wang, S. Z., and Zhang, X. "An immersed boundary method based on discrete stream function formulation for two- and three-dimensional incompressible flows," Journal of Computational Physics Vol. 230, No. 9, 2011, pp. 3479-3499.

${ }^{27}$ Taira, K., and Colonius, T. I. M. "Three-dimensional flows around low-aspect-ratio flat-plate wings at low Reynolds numbers," Journal Of Fluid Mechanics Vol. 623, 2009, p. 187.

${ }^{28}$ Yarusevych, S., and Boutilier, M. S. H. "Vortex Shedding of an Airfoil at Low Reynolds Numbers," Aiaa Journal Vol. 49, No. 10, 2011, pp. 2221-2227.

${ }^{29}$ Taylor, G. K., Nudds, R. L., and Thomas, A. L. R. "Flying and swimming animals cruise at a Strouhal number tuned for high power efficiency," Nature Vol. 425, No. 6959, 2003, pp. 707-711.

${ }^{30}$ McCormick, B. W. Aerodynamics, aeronautics, and flight mechanics: Wiley, 1995. 